\title{
CCARHUAYO, UNA COMUNIDAD QUECHUA EN CUZCO, SU LUCHA POR DEFENDERSE EN EL MUNDO MODERNO
}

\author{
CCARHUAYO, A COMMUNITY QUECHUA IN CUZCO, THEIR STRUGGLE \\ IN THE MODERN WORLD
}

\author{
Leif Korsbaek $^{*}$
}

\begin{abstract}
El texto contiene una descripción etnográfica de la institución conocida como la ronda campesina en la comunidad de hablantes de quechua de Ccarhuayo en le región de Cusco en el sur del Perú.

Se presentan los rasgos generales de la ronda campesina, se da información acerca del surgimiento y el desarrollo histórico de la institución, y se presentan los diferentes tipos de rondas campesinas que existen en diversas regiones del Perú.

De Ccarhuayo se describe su base económica, la escuela y la vida cotidiana, y la ronda campesina de la comunidad se presenta como la institución que expresa la identidad étnica y encarna el derecho comunitario y defiende la comunidad contra los peligros de la modernización, tal como estos peligros se manifiestan, entre otros factores, en la construcción de la carretera interoceánica.
\end{abstract}

Palabras claves: Comunidad, instituciones de la comunidad, modernización, identidad étnica, derecho comunitario.

The text contains an ethnographic description of the institution known as the "ronda campesina" in the Quechua-speaking community of Ccarhuayo in the region of Cusco in Southern Peru.

The general features of the ronda campesina are presented, some information of the origin and the historical development of this institution are presented, together with a list of different types of rondas campesinas that are found throughout the various regions of Peru.

The economic basis, the school and some features of the daily life in Ccarhuayo are described, and the community's ronda campesina is presented as the institution that gives expression to the community's ethnic identity and the community justice system, and protects the community against the dangers of the process of modernization, a process that manifests itself in the construction of the Interoceanic Highway.

Key words: Community, community institutions, modernization, ethnic identity, community right.

\section{Introducción}

A partir de 1492, cuando Cristóbal Colón por casualidad tropezó con un nuevo doble continente y este fuera "integrado" como parte del mundo, el mundo es auténtica y realmente uno y ninguna parte queda aislada, sin articulación con las demás partes. Este "descubrimiento" y la siguiente integración de enormes regiones llevó a la conformación de lo que podemos llamar el "mundo moderno", un mundo en su momento dominado por Europa, con grandes poblaciones de pueblos originarios subyugados a la dominación política desde el "centro", desde Europa.

El desarrollo europeo, que en aquel momento llevaba la batuta, incluía tres procesos paralelos que en su conjunto iban a definir la dinámica del centro y, mediante la dominación, también de la periferia: el proceso de descubrimiento e integración de todas las partes del globo, un proceso que produciría un mundo moderno integrado; el proceso conocido como el Renacimiento, que produciría una nueva sensibilidad que permea la existencia humana de mil maneras y en todos los aspectos, en un nuevo individualismo, una nueva religiosidad y una nueva relación con la naturaleza, para mencionar solamente las más visibles; y el proceso de nacimiento del capitalismo, que produciría una nueva racionalidad económica y una nueva relación entre la economía y la política.

La integración de estos pueblos originarios siempre se ha llevado a cabo en las condiciones del centro, tomando en cuenta sus deseos y necesidades cuando se han rebelado y en una situación altamente asimétrica. Los pueblos originarios insisten en existir de acuerdo con sus culturas tradicionales, organizándose internamente a base de sus estructuras sociales tradicionales y rigiéndose por sus sistemas de derecho tradicionales, una insistencia que los condena a vivir en una permanente posición de defensa contra el mundo moderno que insiste en asimilarlos.

* Escuela Nacional de Antropología e Historia, Instituto Nacional de Antropología e Historia. México. Correo electrónico: leifkorsbaek1941@gmail.com 
La vida de los pueblos originarios se lleva a cabo en el marco de la comunidad, rodeada de las fuerzas del mundo moderno que se manifiestan de mil maneras, y la comunidad y el mundo moderno son entes incompatibles que, sin embargo, tienen que coexistir. La comunidad y las entidades del mundo moderno se insertan de modos diferentes en el mundo, como señaló Carlos Marx hace muchos años: "en el mundo tradicional, la ciudad se incrusta en el campo, en el mundo moderno, el campo es como una extensión de la ciudad". Ya que estos pueblos originarios y sus comunidades viven en una constante posición de defensa, han tenido que desarrollar toda una estructura de defensa, orientada contra la voracidad del mundo moderno que los rodea a todos lados ${ }^{1}$.

\section{La ronda campesina}

La ronda campesina es, antes que nada, una institución que pertenece a la comunidad campesina o indígena: "son órganos de vigilancia y protección propia de las comunidades campesinas; su función es cautelar la vida, la integridad y el patrimonio de los comuneros frente a la existencia de posibles actos que vulneren sus derechos fundamentales"2. Según otro investigador, "son organizaciones de campesinos que en forma voluntaria realizan labores comunales de seguridad de sus pueblos, resuelven pacíficamente los conflictos en sus comunidades y participan activamente en el desarrollo de sus localidades"3. Mientras que para la Defensoría del Pueblo "las rondas campesinas son formas de organización comunal y campesina, que representan y organizan la vida comunal, ejercen funciones de justicia, interlocución con el Estado y realizan tareas de desarrollo, seguridad y paz comunal, dentro de su ámbito territorial" 4 .

Es sabido que las primeras rondas surgieron en Chota, en Cajamarca, en el norte del Perú, en 1976, y se difundieron como un incendio en la pradera, hasta llegar a números increíbles, arriba de 100.000 ronderos, tan solo en la provincia de Cajamarca. Más precisamente, "la primera ronda se fundó en Cuyumalca, estancia contigua a la ciudad de Chota, a las 2 p. m. del 29 de diciembre de 1976. Esta histórica decisión la tomaron los padres de familia de la escuela, y fue ratificada en días siguientes por todas las familias de Cuyumalca. El nombre original fue Rondas Nocturnas" ${ }^{\text {, como se desprende del }}$ acta histórica de la fundación que reza como sigue:
"En la estancia de Cuyumalca, siendo las 2:00 pm. del día veintinueve de diciembre de mil novecientos setenta seis, reunidos los ciudadanos de dicha comunidad, luego de intercambio de ideas se llegó al acuerdo de organizar 'Rondas Nocturnas' para defender los intereses del centro educativo y de toda la comunidad a consecuencia de los continuos robos que se vienen suscitando en agravio de dicho centro y de algunos vecinos. Esta acta tiene la finalidad de organizar a la comunidad y solicitar la licencia respectiva a fin de que sea posible comprar sus armas.

El encargado de organizar las rondas será el Teniente Gobernador, quien previo empadronamiento de la ciudadanía, distribuirá el personal; será también el encargado de seguir los trámites correspondientes para el buen desempeño de sus funciones.

Con lo que se dio por terminado el acta siendo las 3:15 pm.

Copia de la presente acta será remitida a las autoridades respectivas de la provincia a fin de solicitar garantías y parejas de Guardias Civiles cuando el caso lo requiera. Asimismo, la comunidad acordó dirigirse, mediante un memorial, al Presidente de la Corte Superior de Justicia de Lambayeque pidiendo el nombramiento de un juez único de primera nominación por intermedio del juez instructor de nuestra provincia".

Lo anterior es el momento de nacimiento de la ronda campesina, su inicio histórico para así decirlo, pero quedándonos en la dimensión histórica podemos descubrir algunos antecedentes de esta institución: según algunos, "en las rondas parecen confluir tres vertientes institucionales: las guardias de las haciendas por su función, la comunidad campesina, por su organización, y el servicio militar obligatorio, como requisito para ejercer algunos roles", según otros, "es posible afirmar que no existe sino un solo fenómeno -a diferencia de lo que creen algunos estudiosos de las rondas- que se puede considerar como antecedente de esta organización: las guardias campesinas de las haciendas, encargadas por el hacendado de vigilar su propiedad y de perseguir a los abigeos"'.

"Una serie de factores confluyen en el surgimiento de las rondas campesinas. La crisis económica, el 
abigeato, la corrupción de la justicia y el vacío de autoridad estatal aparecen mencionados de modo prácticamente unánime en los estudios"7, mientras que Orin Starn añade otra razón: "la recompensa cultural que en el campo norteño tiene el ser rudo, terco y temerario" 8 .

Es evidente que en tal cantidad de instituciones que existen en regiones muy alejadas y de muy diferente carácter y con trayectorias históricas muy diversas, no todas las rondas pueden ser idénticas, deben de existir diferentes tipos de rondas campesinas, lo que sí es evidente al caso, y podemos inicialmente distinguir cuatro tipos de rondas campesinas ${ }^{9}$.

"Un primer tipo de rondas campesinas son aquellas surgidas sobre la base de los caseríos, donde no han existido comunidades campesinas, como en el caso de Cajamarca, San Martín y Amazonas fundamentalmente. Un segundo tipo son las que han surgido dentro de las comunidades campesinas, como es el caso en Piura, Ancash, La Libertad y en el sur andino (casi todas las rondas campesinas de Carabaya en Puno y de las provincias de Espinar y Canas y del distrito de Quispicanchis en Cusco), que cumplen el papel de órgano auxiliar de las comunidades y dependen de estas. Un tercer tipo son aquellas rondas campesinas que existen en comunidades nativas de la selva peruana. $Y$ finalmente, un cuarto tipo son los denominados 'comités de autodefensa' que nacieron en la década de los noventa impulsados por el Estado para la lucha contra la subversión en la época de la violencia política, y que han surgido indistintamente al interior de las comunidades campesinas y parcelas o caseríos donde no hay presencia de comunidades campesinas"10.

- El primer tipo de rondas campesinas, aquellas que existen en los caseríos, que "surgen en una región abandonada por el Estado. Esta ausencia, sin embargo, no es ninguna peculiaridad de la sierra norte. Lo peculiar en la sierra norteña es más bien que los campesinos no contaron con mecanismos sociales propios para contrarrestar este vacío de autoridad y sus consecuencias. La ausencia de un organismo regulador se manifestó sobre todo en la vida cotidiana; la región era afamada por peleas sangrientas, hurtos de ganado, que aumentaron después del retiro de los hacendados" 11 . No me parece que se trate de "una región abandonada por el Estado", pues por un lado se trata de un proceso histórico que ha producido una situación en donde "el departamento de Cajamarca, como toda la sierra norteña del Perú, es tierra de campesinos parcelarios", y "en Cajamarca el número de comuneros, cuyas tierras oficialmente son propiedad de la comunidad en su conjunto, apenas llega al 0,6\% de la población campesina"12, por otro lado, un gran impulso que contribuyó a la producción de esta situación, lo tenemos que buscar en la reforma agrario de Velasco Alvarado, como señala Carlos Iván Degregori: "las rondas de Cajamarca y Piura surgieron teniendo como telón de fondo la desaparición de los terratenientes luego de la reforma agraria, y la multiplicación de los parcelarios libres. En Piura, la propiedad individual se encontraba revestida bajo la etiqueta de comunidad campesina (Pérez, 1992; Huber, 1995), pero esta era más una formalidad, sin los lazos de ayuda mutua, trabajo colectivo y asambleas que suelen caracterizar como tipo ideal a las comunidades de los Andes del Sur. En este contexto, la organización rondera logró desarrollar una sólida identidad colectiva en la que muchos encontraron similitudes con las comunidades campesinas de otras partes de la sierra"13. Resumiendo, en una parte de Cajamarca, exactamente donde surgió en 1976 la primera ronda campesina, tenemos una situación en la que no existe la tenencia comunal de la tierra, donde los campesinos son parceleros y no existe una larga tradición de una toma de decisiones colectivas; en este ambiente la ronda llega a llenar un vacío político y constituirá algo así como una estructura social de la comunidad campesina.

- El segundo tipo surge en regiones donde las condiciones son radicalmente diferentes, donde los campesinos viven en comunidades con una sólida tradición colectiva que se enmarca en un contexto de tenencia comunal de la tierra: "el III Censo Nacional Agropecuario de 1994 revela que en el departamento de Apurímac existían 438 comunidades campesinas registradas que poseían 1.228.711 hectáreas de tierra y la mayor parte de dicha población rural vivía en estas unidades de propiedad comunal de la tierra (81\% en Apurímac)"14, de manera que "las rondas campesinas del sur andino, en tanto organizaciones distintas a las que existen en el resto del país, así como por haberse desarrollado en un contexto político, 
social, histórico e incluso cultural, diferente a las rondas del norte y centro, podrían definirse como órganos de vigilancia, protección y justicia de las comunidades campesinas, ayllus, centros poblacionales y parcialidades, para ejercer funciones relacionadas a la seguridad comunal, justicia comunal restaurativa, promover el desarrollo comunal, y establecer relaciones de coordinación y diálogo con las instancias del Estado, dentro del ámbito territorial donde se organizan, conforme a los valores, principios y creencias de su propia lógica y racionalidad ${ }^{15}$.

- El tercer tipo, "las rondas campesinas de comunidades nativas de la selva peruana"16, donde en muchos casos han tenido que coexistir con el narcotráfico y otras actividades ilícitas que existen debido a la casi total ausencia del Estado y de sus representantes. Una descripción romántica de la ausencia del Estado en la selva amazónica es la famosa novela Pantaleón y las visitadoras, de Mario Vargas Llosa, un excelente novelista y un miserable pensador político; una impresión mucho menos romántica de la ausencia del Estado y de cualquier normatividad en la selva amazónica tiene uno en la situación en las orillas de la carretera de Cusco a Puerto Maldonado, con la explotación despiadada en las minas de oro y la prostitución infantil en los campamentos ${ }^{17}$ (Figura 1).

- El cuarto tipo, los denominados "comités de autodefensa" es probablemente el más problemático, como se señala en el informe final de la Comisión de Verdad: "En ningún otro actor de la guerra, la línea divisora entre perpetrador y víctima entre héroe y villano, es tan delgada y porosa como en los comités

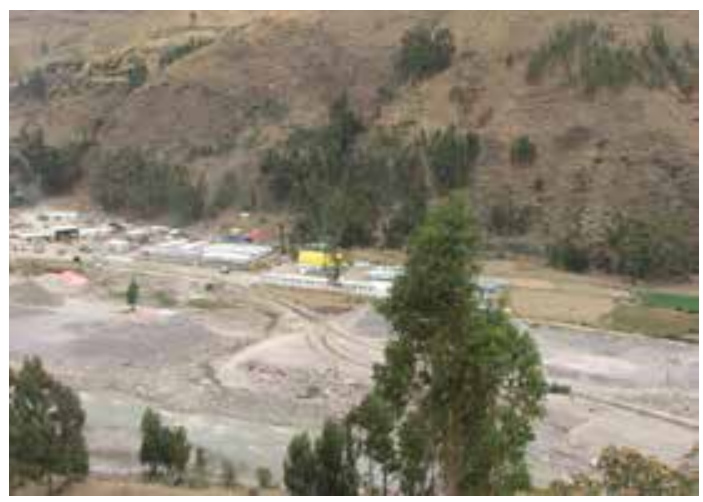

Figura 1. El campamento de construcción de la carretera interoceánica. de autodefensa (CAD) o rondas campesinas contrasubversivas" 18 .

Podemos buscar los antecedentes del modelo ${ }^{19}$ en la guerra de liberación en la colonia británica de Malasia de 1948 a 1960, en pleno proceso de descolonización: "durante esta guerra nace también la doctrina militar de las aldeas estratégicas, como fuera bautizada más tarde en Argelia y Vietnam y que consiste, ante todo, en desplazar las poblaciones que suministran información y alimentos al enemigo, para privarlo de su base de suministros y obligarlo a salir de la selva. Igualmente se inflingen castigos colectivos a las aldeas cuyos habitantes sean sospechosos de ayudar a los rebeldes, secuestrando a sus habitantes y a veces llegando incluso a matarlos de hambre reduciéndoles sus raciones de arroz. El plan inicial, llamado Plan Briggs, preveía el desplazamiento de aproximadamente 500.000 habitantes de aldeas retiradas en su mayoría de origen chino, hacia nuevas aldeas rodeadas de alambradas y estrechamente vigiladas. Esto dará sus frutos a partir de 1951, al debilitar de manera significativa las fuerzas insurgentes Por otra parte, las fuerzas británicas encarcelan a 34.000 personas durante los primeros ocho años del estado de emergencia"20.

Los norteamericanos adoptaron el modelo en su guerra etnocida en Vietnam: "los norteamericanos se aprestaron a construir varias aldeas estratégicas para concentrar a la población vietnamita con el fin de reagruparla y controlarla, a la vez que se le cortaba al Vietcong sus áreas cotidianas de aprovisionamiento y apoyo logístico. A fines de 1962 los americanos habían levantado ya unas 3.500 aldeas estratégicas, así como unas 2.000 más en los siguientes años. En estas aldeas sus habitantes podían elegir sus propios consejos municipales y demás estructuras administrativas simples"21.

Finalmente lo encontramos en el periodo de violencia en Guatemala, entre 1978 y 1983: "Guatemala, la población, los pueblos indígenas, así como la comunidad internacional, fueron sacudidos durante mayo del presente año por la noticia no sorprendente, pero sí abominable y repudiable por la decisión del Tribunal de Sentencia y demás tribunales de justicia del departamento del Quiche, al haber absuelto de sus cargos al genocida y etnocida Cándido Noriega Estrada. Cándido Noriega es responsable de masacres, asesinatos, torturas, violaciones, robos, amenazas e intimidaciones contra los indígenas de Chiche y Chinique del departamento del Quiche. Sus delitos suman 35 asesinatos, 44 secuestros, 14 violaciones, 
10 robos agravados, 2 hurtos agravados, 7 incendios, 6 amenazas de muertes, 9 daños físicos, 23 detenciones ilegales, 6 lesiones leves y allanamientos, acumulando 156 delitos. Noriega es un excomisionado militar, vecino y originario del municipio de Chinique de las Flores del departamento del Quiche. Su origen ladino pobre, pero con nivel de vida por encima del de la población que habita la región. Él vive en un área donde el $98 \%$ de la población es Maya-K'iche. Durante los años de recrudecimiento de la persecusión del ejército hacia las comunidades y sus pobladores, años de 1978 a 1985, la institución armada generó una serie de mecanismos para acabar con poblaciones enteras. Concentró todos sus esfuerzos para cometer genocidio y etnocidio, porque todos los hechos más atroces de nuestra historia reciente se cometió en los lugares donde se asienta el pueblo Mam, Kaqchikel, Q'eq'chi, Achi, Tzutujil, Poqomchi, K'iche, entre otros. Los estrategas del ejército instituyeron las Patrullas de Autodefensa Civil y comisionados militares como agentes directos de represión; el ejército impulsó la contratación de personas informantes conocidos en nuestro país como orejas, formó los polos de desarrollo, aldeas modelo y aldeas estratégicas o puntos de concentración, con el supuesto de acabar con un movimiento guerrillero emergente, pero cuyo fondo era terminar con los sistemas político, económico, social y cultural de las raíces de miles de años de existencia del pueblo Maya. Dentro de ese contexto se ubica la actuación de Cándido Noriega Estrada, porque solo así puede comprender por qué ha sido absuelto de los hechos que han causado tanto dolor y destrucción, no solo física sino psicológica en los indígenas, con resultados y daños irreversibles en la etapa de conciliación comunal y municipal que estamos emprendiendo después de la firma de la paz firme y duradera. También dentro de este marco general se puede entender el poder que tienen todavía los militares a través de jueces como Olegario Labé Morales, quien a pesar de ser cuestionado desde hace muchos años por su complicidad con los militares, aún sigue sin ser destituido; y es precisamente él el artífice principal para darle la libertad a uno de los asesinos de nuestro pueblo" 22 .

Volviendo al Perú, "para 1993 el éxito de los Comités de Autodefensa era más que notable por su satisfactoria respuesta a las necesidades de seguridad de las comunidades, y también por su protagonismo en la organización política de las localidades (Cuadro 1). El siguiente cuadro muestra la distribución e importancia numérica de estos grupos"23.
Cuadro 1

\begin{tabular}{rlrrr}
\hline $\mathrm{N}^{\circ}$ & Departamento & CAD & Integrantes & Armas \\
\hline 1 & Tumbes & 70 & 2.871 & \\
2 & Piura & 282 & 17.778 & \\
3 & Lambayeque & 105 & 5.730 & 194 \\
4 & Cajamarca & 443 & 27.927 & 916 \\
5 & La Libertad & 219 & 14.018 & 858 \\
6 & Ancash & 27 & 1.350 & 142 \\
7 & Junín & 525 & 34.537 & 3.855 \\
8 & Cerro de Paso & 68 & 6.072 & 626 \\
9 & Ayacucho & 1.564 & 61.450 & 5.583 \\
10 & Huancavelica & 198 & 10.658 & 646 \\
11 & Lima & 33 & 2.869 & 508 \\
12 & Puno & 86 & 3.627 & 160 \\
13 & Loreto & 30 & 911 & \\
14 & Amazonas & 112 & 8.295 & 260 \\
15 & Apurímac & 63 & 3.618 & 100 \\
16 & Cusco & 106 & 5.802 & 440 \\
17 & Madre de Dios & 20 & 1.232 & 60 \\
18 & Huanuco & 163 & 13.578 & 868 \\
19 & San Martín & 55 & 11.658 & 892 \\
20 & Ucayali & 36 & 1.286 & 170 \\
& Total & 4.205 & 235.465 & 16.196 \\
\hline
\end{tabular}

\section{Ccarhuayo: el distrito y la comunidad}

La comunidad de Ccarhuayo es la capital del distrito de Ccarhuayo que se encuentra en la provincia de Quispicanchis en el centro de la región de Cuzco, en el extremo sur del Perú ${ }^{4}$ (Figura 2).

La cuenca más importante de la provincia de Quispicanchis está formada por el río Vilcanota y por su margen derecho recibe las aguas de los ríos pequeños como Tintinco, Thiomayo, Pampachulla, Cachimayo, Huaraypata en Quehuar. Otra cuenca importante es la formada por el río Mapacho que por su ubicación geográfica y debido a su altura de $3.700 \mathrm{msnm}$ no es tan aprovechada por la agricultura, siendo rico en pesca de trucha. Finalmente la última cuenca discurre hacia la vertiente oriental formando la cuenca hidrográfica de Marcapata.

CLIMA: esta provincia tiene una variedad de climas como son: frígido, templado, seco, y cálido húmedo por existir diferentes pisos ecológicos .

En la zona intermedia, la temperatura promedio anual máxima es de $13,6-15,5^{\circ} \mathrm{C}$, mientras que en la zona altoandina encontramos una temperatura promedio anual máximo de $8,1-4,5^{\circ} \mathrm{C}$.

El distrito de Ccarhuayo abarca, aparte de la cabecera, doce comunidades y cuatro anexos, con 2.614 habitantes, distribuidos como sigue ${ }^{25}$ (Cuadro 2). 


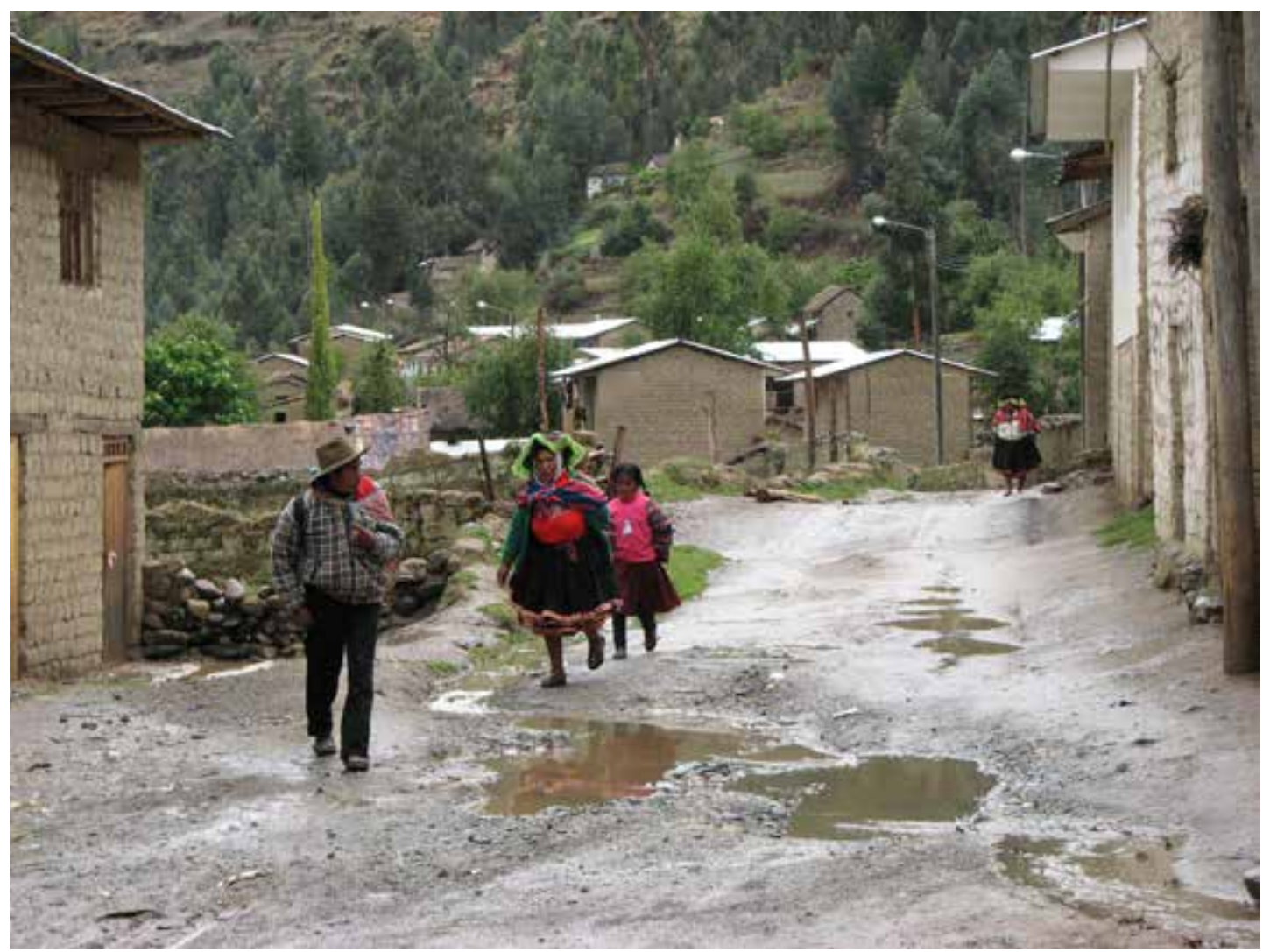

Figura 2. Una calle en Ccarhuayo.

Cuadro 2.

\begin{tabular}{|c|c|c|c|c|c|c|c|c|c|c|}
\hline Comunidad & Anexo & $<1$ Año & 1 Año & 2-4 Año & 5-9 Año & 10-19 Año & 20-49 Año & 50-59 Año & >80 Año & Total \\
\hline Ccarhuayo & & 17 & 7 & 50 & 67 & 153 & 172 & 26 & 30 & 522 \\
\hline Anccasi & Central & 6 & 4 & 8 & 15 & 25 & 34 & 1 & 6 & 99 \\
\hline Anccasi & Lawani & 5 & 1 & 3 & 17 & 35 & 39 & 12 & 6 & 118 \\
\hline Anccasi & Pirki & 3 & 4 & 10 & 18 & 25 & 37 & 9 & 7 & 113 \\
\hline Ccasapata & & 2 & 3 & 15 & 15 & 28 & 38 & 8 & 14 & 123 \\
\hline Ccasapata & Eukora & - & 1 & 3 & 2 & 10 & 11 & 5 & 5 & 37 \\
\hline Chillihuani & & 2 & 6 & 15 & 16 & 31 & 46 & 1 & 16 & 133 \\
\hline Chillihuani & Junuta & - & 4 & 8 & 9 & 22 & 27 & 3 & 4 & 77 \\
\hline Chuclluhuiri & & 3 & 7 & 14 & 25 & 31 & 52 & 11 & 16 & 159 \\
\hline Cjallhua & & 1 & 5 & 20 & 34 & 44 & 67 & 7 & 13 & 191 \\
\hline Ccoya & & 3 & 6 & 15 & 24 & 34 & 43 & 5 & 9 & 139 \\
\hline J.C. Mariategui & & - & - & 2 & 11 & 34 & 22 & 4 & 7 & 80 \\
\hline Marcjupata & & 2 & - & 2 & 8 & 10 & 14 & 2 & 5 & 43 \\
\hline Hachaccalla & & 5 & 6 & 19 & 32 & 51 & 65 & 10 & 7 & 195 \\
\hline Parcoccalla & & 6 & 2 & 17 & 25 & 53 & 86 & 11 & 9 & 209 \\
\hline Sumana & & 4 & 5 & 15 & 42 & 42 & 64 & 12 & 10 & 194 \\
\hline Tayankani & & 4 & 4 & 9 & 30 & 45 & 70 & 1 & 19 & 182 \\
\hline Total & & 63 & 65 & 225 & 390 & 673 & 887 & 128 & 183 & 2.614 \\
\hline
\end{tabular}


Aquí será necesario intercalar dos comentarios acerca de la región y la comunidad como conceptos y como universos de estudio.

En primer lugar, en otra ocasión señalé que "las instituciones comunitarias cambian bajo el impacto del neoliberalismo, una transforrmación que se ejemplifica en el cambio de la institución conocida como el sistema de cargos en otra institución mucho más militante, explícitamente de defensa de la comunidad: la ronda campesina en el Perú, y sus equivalentes en otras partes del mundo" 26 , un cambio que se manifiesta en México como la transformación del sistema de cargos en la policía comunitaria, mientras que en el Perú el resultado es la ronda campesina.

En segundo lugar, partiendo del estudio del sistema de cargos, la primera observación acerca de esta institución es que es posiblemente la institución que se caracterice más netamente como una institución de la comunidad.

Perú es un país de comunidades campesinas, hasta tal grado que la comunidad "no es solo la institución más antigua sino la institución más importante -en términos sociales y demográficos-que existe en el país" ${ }^{27}$, una importancia que se expresa cuantitativa y empíricamente en la existencia de 5.680 comunidades campesinas, con una población de 2.700 .000 personas, que corresponde al $50 \%$ de la población rural, y el $15 \%$ de la población total en la cordillera andina, "la región más pobre del Perú"28, y es en este universo que nació y se desarrolló la ronda campesina.

El concepto de "comunidad" es uno de los términos clave de la antropología, y entre antropólogos por lo regular se piensa que el concepto de "comunidad" esté bien definido, pero no es así ${ }^{29}$. Para los fines de la presente investigación una comunidad debe poseer seis características: 1) es un proceso, 2) llevado a cabo por una colectividad, 3) frecuentemente pero no siempre dentro de un marco territorial, que 4) tiene coherencia horizontal, 5) coherencia vertical, 6) y tiene historia ${ }^{30}$.

Política y administrativamente el Distrito de Ccarhuayo se encuentra representado de la siguiente manera: un alcalde y cinco regidores con sus respectivos cargos que rotan cada año en sus funciones, cada regidor entra en un sorteo de cargos para un año. Un gobernador que es nombrado por el ministerio interior de justicia, y también existe en cada comunidad un teniente gobernador.

\section{Cultivos en Ccarhuayo}

En las comunidades del distrito de Ccarhuayo utilizan dos espacios bien definidos de cultivos, de acuerdo con la altitud.

Región quechua y suni (conocido por los lugareños como pampa). Este piso se ubica a partir de 3.300 a $3.800 \mathrm{msnm}$, siendo esta zona baja y plana con mayores aptitudes para la producción agropecuaria: maíz, papa, zanahoria, cebolla , ajo, perejil y alfalfa. También cuenta con algunos animales como vacas chuscas o criollas y estabuladas, ovejas, caballos, burros y cuyes. Asimismo, cuentan con algunos frutales como duraznero, manzanos, capulí, tintin, saúco, pera y tumbo (Figura 3).

Puna o jalca (conocida como orqo) ubicado entre los 3.300 a $4.000 \mathrm{msnm}$, posee pendientes bien pronunciadas y una topografía accidentada con procesos de erosión, la agricultura es en secano, principalmente apto para el cultivo de papa, oca, olluco, mashua, quinuna, cebada y tarwi.

Puna alto (es el piso ecológico que se ubica por encima de los $4.000 \mathrm{msnm}$ ), parte de su relieve está conformado por mesetas aptas para la crianza de auquénidos (alpaca, llama en pequeñas cantidades (con tendencia a desaparecer) por la presencia de ovejas en grandes cantidades, algunos caballos y vacas.

Desde la conquista española hasta nuestros días el pueblo originario en el Perú sufre una agresión alimentaria desde el punto de vista económico y cultural. La respuesta popular a esta agresión ha significado la supervivencia de hábitos y esquemas alimentarios nativos; el mejor ejemplo es el masivo consumo de la coca por las personas más ancianas. En el distrito de Ccarhuayo y sus comunidades, su dieta está basada en el consumo de granos andinos

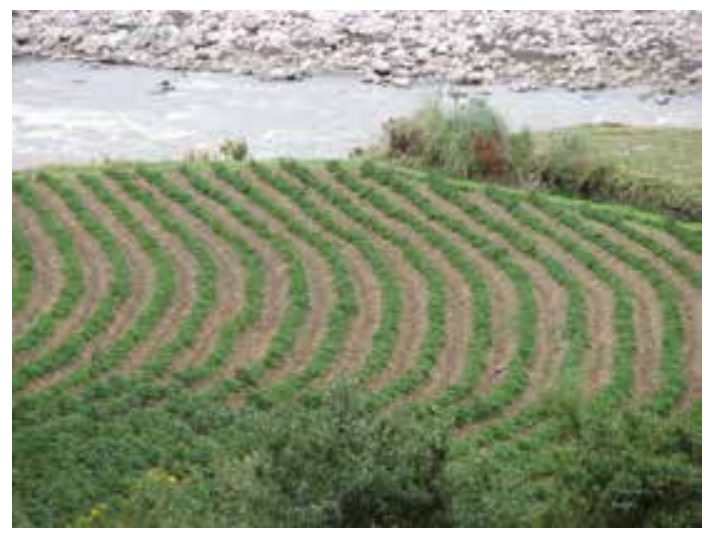

Figura 3. Una chacra (milpa) al lado del río. 
como tarwi, quinua, habas, oqa, maíz, año, lisas (olluco), cebada y yuy (nabo); chuño y moraya son papas desecadas para consumo en una temperatura muy baja.

El alimento de muchas familias típicas de comunidades es: por la mañana, una sopa hecha de chuño "chuño lagua con un poquito de carne y charqui; si no hay carne, solo así, no más", y tostada de maíz (hanqa) y su mate de panti o alguna hierba del lugar, bueno, si hay azúcar ${ }^{31}$.

Por lo normal no cocinan para el medio día, porque a esa hora están pastoreando sus animales o están trabajando en sus chacras. Para ello se llevan su qoqaho que consta de chuño fase con un poquito de sal, ají o charqui, más año, oqa o mote hasta en la tarde.

Al regresar al hogar, como a las 6 o 7 p.m. se cocinan un poco de sopa, ya sea de lisas o solamente mate con papas sancochadas.

Por lo general, durante la semana comen sus productos que han cosechado y que tienen en su taque o reserva que posee cada familia en su hogar.

Bueno, los domingos y los días festivos visitan a alguien importante, celebran cumpleaños, matan a sus animales como cuyes, gallinas, ovejas, que se comen acompañados con fideo, arroz, que le llaman comida de lujo o de mistis.

Los domingos para la familia significa comer frutas, manzanas, pepino, plátano, uva, pan, gaseosa, galletas, dulces, comer pescado frito con arroz, segundo de fideos, gelatina y de paso comprar un poco de azúcar, sal, arroz, fideos y verduras como zanahoria y cebolla.

\section{La escuela en Ccarhuayo}

En la cabecera hay un colegio (escuela secundaria) y una escuela primaria, y en las comunidades solo hay planteles de educación primaria. Tanto en la escuela primaria como en la secundaria la casi totalidad de los profesores provienen de otras comunidades y otras regiones.

Las materias que se enseñan son materias que tienen utilidad para el mundo moderno, muy lejos de los intereses, las tradiciones y las necesidades de la comunidad tradicional, y todo el funcionamiento de la escuela refuerza la impresión de que el aparato educacional viene de fuera y es impuesto a la comunidad.

El uso respectivo del quechua y del español en la escuela primaria es muy claro: el español es la lengua "legítima", en el sentido de que toda instrucción, explicación y corrección se transmite en español, mientras que la lengua franca entre los alumnos es el quechua, de manera que el quechua, la lengua materna de los alumnos inevitablemente se convierte en una especie de lengua subterránea, que se usa solamente en contextos y situaciones marginales, a veces ilegales secretos.

Por un lado es cierto que la escuela definitivamente no pertenece a la comunidad, se siente en todo como algo postizo o bien impuesto, pero por otro lado es también cierto que la escuela se relaciona íntimamente con la familia y, a base de un cuestionario aplicado a 129 alumnos en el colegio, se ha calculado que el tamaño medio de la familia en Ccarhuayo es de 6,7 personas, y es típicamente una familia nuclear, en donde el jefe de familia es el padre.

En la comunidad nos sentimos como en "otro mundo", en el mundo tradicional, muy claramente separado del mundo moderno que representa la escuela. En el mundo moderno al que pertenece la escuela encontramos también otras escuelas, las universidades. A este nivel de la educación superior la relación con la comunidad es también tenue, de la comunidad solamente dos jóvenes cursan educación superior en la Universidad en Cusco.

\section{La ronda campesina en Ccarhuayo}

Como también en otras partes de la región donde se encuentra Ccarhuayo, la ronda campesina nació un poco después de 1991 y, según la tradición oral, por iniciativa del padre Antonio, un español que lleva muchos años residiendo allá y ha logrado conquistarse un lugar en la sociedad, antes que nada políticamente. Más precisamente,

"los líderes comuneros con el apoyo del sacerdote Antonio concretaron la idea de constituir una central de rondas en los distritos de Ocongate y Ccarhuayo, así que esta organización se constituyó el 20 de agosto de 1992, en el sector denominado Yanacocha, en esta reunión participaron un promedio de 6.000 comuneros, y ese mismo día se procedió a denunciar aproximadamente a treinta abigeos con pruebas de delitos cometidos. La ronda campesina de Ocongate-Ccarhuayo aglutinaba a 46 comunidades, encontrándose entre ellas también comunidades del distrito de Ccatca 
(Nueva Esperanza Ccapana, Asociación Ccapana y Andayaque)".

Las rondas campesinas de Quispicanchi nacieron como asociaciones de autodefensa frente al abigeato y otras faltas menores, y hoy tienen una antigüedad aproximada de veinte años (mediante sus diversos comités comunales de rondas campesinas y posteriormente a las centrales distritales y zonales).

El motivo para crear una ronda campesina aquí fue el mismo que en otras partes: "a finales de los años ochenta e inicios de los años noventa la fuerte presencia de abigeos, que despojaban a los campesinos de sus bienes (alimentos, ropa y otros). Justificaron la necesidad de constituir una Central Distrital de Rondas Campesinas".

Es significativo que "contrariamente a otros lugares la presencia de Sendero Luminosa en la zona no era muy intensa, podemos mencionar como referencia que en 1986 y 1987 en el sector de Hualluahuallhua se produjeron robos atribuidos a Sendero Luminoso, estos delitos tenían un gran impacto en la zona", sin embargo, "luego de ser capturados los culpables por la policía, se advirtió que no eran senderistas sino delincuentes comunes".

"La iniciativa por difundir el trabajo de las rondas surge de dos fuentes. En 1990 el sacerdote Antonio de la parroquia de Ocongate a solicitud del catequista Mariano Chillihuani trajo libros de Cajamarca sobre las rondas campesinas para que los catequistas se encargasen de difundirlos en las comunidades. Un año después, el sacerdote Antonio en coordinación con una ONG, CCAIJO, organizó un curso respecto de la experiencia de las rondas en Cajamarca. Paralelamente, algunos líderes de la zona asistieron desde fines de los años ochenta a encuentros de rondas campesinas en la provincia de Quispicanchis. Según Vidal Fuentes, exdirectivo y miembro fundador de la ronda, él mismo fue víctima de un robo de 20 llamas y los otros han sido testigos de casos similares. En esta época la situación era tan grave que los comuneros no guardaban sus bienes en sus casas, sino en las poblaciones cercanas, por temor a ser víctimas de los abigeos, por ejemplo las dejaban donde un conocido en la capital del distrito".
Años después, en 1995, surgieron nuevas fuerzas políticas que exigieron que se desmembrara la Central de Ocongate (Figura 4) por razones de proximidad geográfica y rivalidad gerencial, pues se había constituido una nueva central distrital en Ccarhuayo, pero por agentes externos se mantenía todavía la relación de dependencia de organización. Un factor importante es que la Central había sufrido un debilitamiento en su interior, pues las rondas en esta zona no habían logrado articularse y definir con claridad cuáles eran sus atribuciones y responsabilidades, y se dio énfasis en la necesidad de cumplir con los trámites legales necesarios y la elaboración de un estatuto interno.

El origen de esta ronda resultó del interés de los pobladores, las experiencias originarias y no originarias de la zona, y en su gestión están notablemente presentes los elementos consuetudinarios ${ }^{32}$. En su relación con la justicia formal, la ronda de Ccarhuayo no ha tenido mayores problemas, más bien en general las autoridades oficiales apoyan el trabajo de la ronda con cierto recelo.

\section{Organización de la ronda de Ccarhuayo}

La ronda campesina en Ccarhuayo se encuentra organizada en dos sectores.

1. Carrhuayo alto, que conforman las comunidades de Anccasi, Hachacalla, Chculluhuiri. Es el primer sector que busca su autonomía por razones de lejanía.

2. Ccarhuayo bajo, sector conformado por las comunidades de Ccjalhu, Sumana, Marjupata, Ccoya, Ccasapata, Chillihuani, Tayancani,

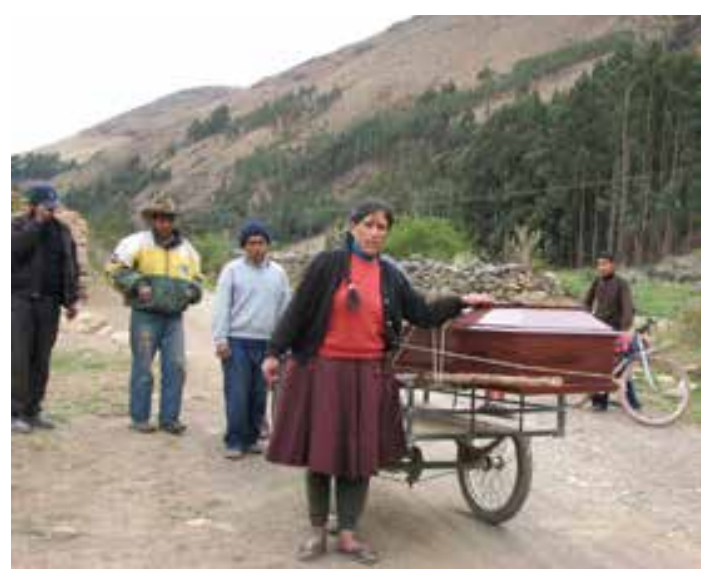

Figura 4. Hacia un entierro en Ocongate. 
Parccocalla y Ccarhuayo, con sus respectivos comités de base, conformando la Ronda Campesina Central de Ccarhuayo.

Es importante mencionar que existe dentro del distrito de Ccarhuayo una empresa minera que paga los trámites de formalización de la organización, haciendo que estos pierdan su neutralidad e imparcialidad respecto de un conflicto que existe entre la empresa y las comunidades de la zona. Es importante resaltar que en la actualidad existen discrepancias muy notorias entre las comunidades de Cjallhua y Parccocalla.

El Comité Central Campesino está conformado por 14 miembros de la ronda y cada uno de ellos son elegidos democráticamente por dos años. El comité tiene un presidente central y un vicepresidente, un secretario de actas, un secretario económico, un secretario de organización y una secretaria de la mujer, y además tiene un vocal, dos jefes mujeres ronderas y dos ronderos.

Un principio de esta organización es la "educación para la prevención", característica que la distancia de otras rondas campesinas e inclusive del mismo Estado, que solo se hace cargo de reprimir y sancionar a los delincuentes.

Además de los casos de abigeo, la ronda trata problemas de violencia familiar, violencia sexual y menores en situación de abandono. Estos casos son tratados de manera interna. Luego se tratan conflictos acerca de tierras entre comunidades y entre comuneros o fiscalización de los funcionarios del distrito. Esta clase de problemas requiere otro tipo de gestión. En general la ronda asume un rol conciliador en la solución del conflicto redactando en sus libros "actas de conciliación" entre las partes.

Con relación a las sanciones que se dan en casos comunes, estas pueden consistir en: baños en una laguna o en un río; latigazos; ejercicios físicos; caminatas desnudos.

Dentro de estas sanciones podemos mencionar:

- Bañarlos en una laguna refiere al "catolicismo andino vigente en las comunidades campesinas de la zona", siendo el agua un elemento purificador.

- El latigazo hace mención a dos elementos: uno es la figura de la "autoridad mayor", que actúa como "un padre que imparte cariño, pero en algunos casos rigor a sus hijos", y se percibe como arte de la "educación para la prevención"; el segundo elemento se ubica dentro del "catolicismo andino vigente" y se da en una ceremonia especial donde la persona que ha de dar los latigazos al ladrón se persigna, para luego pegar solamente tres latigazos que simbolizan a la santísima trinidad (padre, hijo y espíritu santo).

- El ejercicio físico se ha adaptado con el nombre de "reflexión" y proviene de las prácticas del servicio militar de algunos miembros de la ronda campesina.

La ronda tiene vínculos con los siguientes actores: la policía nacional, el juez de paz en Ccarhuayo, quienes son invitados a participar en los encuentros generales, estas autoridades apoyan a la ronda campesina.

En la raíz de la ronda campesina en Ccarhuayo encontramos un evento que ha sido elaborado en un mito fundacional: un año no especificado se vio la región afectada por una pandilla de siete hombres y una mujer que robaron, asaltaron y asesinaron. Finalmente se juntaron los campesinos y rodearon a los ocho malvados, matando a uno, ahuyentando a los demás y tomando presa a la mujer. La mujer no quiso delatar a sus cómplices, así que se acudió al castigo más común en la región: desde un puente la tenían amarrada y la bajaron en el agua del río que, en pleno invierno, está muy, muy fría. La mujer aguantó y solamente la tercera vez que la bajaron al agua helada se rindió y delató a los demás de la pandilla. Un elemento esencial del relato es que los campesinos lograron apresar a los bandidos, mientras que la policía había fracasado. Este patrón se repite en todas las regiones donde existe la ronda campesina -que la ronda funciona, la policía no-y revela en parte una realidad que se encuentra a la raíz del nacimiento de la ronda a partir de 1976: la ineficiencia de la policía.

En Ccarhuayo la ronda campesina ocupa netamente un espacio político, pues mientras que el gobierno local se encuentra en manos del partido del gobierno nacional, el APRA con Alán García en la presidencia, de una orientación neoliberal, la oposición política se organiza dentro de la ronda campesina $^{33}$.

La primera vez que entrevistamos al presidente de la ronda campesina, en 2007, tuvimos la impresión de que su discurso no era el típico discurso de un campesino, ni siquiera de un líder campesino, parecía más bien el de un académico o, por lo menos, una persona citadina con un 
sólido conocimiento de la historia y la política. $\mathrm{Al}$ conocerlo mejor, supimos que era egresado de "La Cantuta", la universidad pedagógica nacional en Chosica, unas dos horas al este de Lima sobre la carretera central y que era un campesino que había aprovechado las posibilidades para ir a vivir unos años con parientes en Lima y terminar una licenciatura en pedagogía ${ }^{34}$, y ahora es una especie de intelectual orgánico en Ccarhuayo.

La fuerza convocatoria de la ronda campesina es impresionante y es un factor político con el que hay que contar. En una asamblea rutinaria de las rondas de 25 comunidades, celebrada arriba de Ccarhuayo, se juntan entre 500 y 1.000 ronderos para discutir la situación y elegir a las nuevas autoridades.

En años recientes, las actividades de la ronda han cambiado un poco de carácter: mientras que anteriormente era una institución de defensa contra el abigeato, hoy, no obstante que sigue la lucha contra los abigeos, tiende a ser una policía moral de la comunidad: la mayor parte de los casos enjuiciados son de hombres y mujeres que han sido sorprendidos engañando a sus cónyuges, que vivan en unión libre, matrimonio civil o matrimonio católico. Las mujeres son castigadas por las mujeres de la ronda campesina, obligadas a correr desnudas por la pequeña plaza de la comunidad, mientras que a los hombres se les aplica el castigo de agua en el río.

En seguida se reproduce un cuestionario que aplicamos al principio de nuestra estancia en Ccarhuayo, como un primer acercamiento a una institución desconocida en un terreno también nuevo y desconocido; el entrevistado, que es de la cabecera, es bastante típico y se destacan algunos detalles de la ronda en Ccarhyuayo, así que sirve como un primer acercamiento al perfil del típico rondero en Ccarhuayo ${ }^{35}$ :

1. ¿Cuál es su nombre y apellido?

Enrique Puma Cutida

2. ¿Cuál es su edad? 44 años

3. ¿Dónde fue usted a la escuela y cuantos años? Ccarhuayo, 4 años

4. ¿Cómo se llama su esposa? Juliana Achahui

5. ¿De dónde es su esposa? Comunidad tayankani

6. ¿Cuántos hijos tiene, cómo se llama y cuál es su edad?

3 hijos de 14 de 12 y 9
7. ¿Cómo es su hogar?

Regular, bien

8. ¿Qué tierras tiene usted?

Temporal y riego

9. ¿De qué vive usted?

Agricultura

10. ¿Usted ha trabajado fuera de la comunidad? Sí

11. ¿Usted ha ocupado cargos religiosos? No

12. ¿Usted ha ocupado cargos civiles? Sí

13. ¿Es usted rondero? Sí

14. ¿Cómo conoció usted la ronda? Participando en la organización de la ronda

15. ¿Tiene usted parientes en la ronda? Sí

16. ¿Cuántos años lleva usted en la ronda? 15 años

17. ¿Por qué usted participa en la ronda? Por la justicia

18. ¿Usted ha participado en alguna acción contra criminales?

No

19. ¿De qué manera?

20. ¿Cuál ha sido el efecto de la ronda, en su opinión?

Bien

21. ¿Cómo se podría mejorar la ronda?

Con la organización

22. ¿Cuál sería la mejor justicia, según usted?

La ronda

\section{Conclusiones}

Para formular unas conclusiones a este trabajo tenemos que regresar a la comunidad Ccarhuayo, y ver las amenazas contra su existencia como comunidad. Pero antes de proceder a formular una conclusión, déjenme presentar los dos puntos fundamentales de mi antropología.

En primer lugar, estoy convencido de la existencia de una lucha prolongada sin cuartel entre dos mundos: el mundo moderno, con su individualismo, y el mundo tradicional, basada en la comunidad como lo dado.

En segundo lugar, también me parece claro que el Estado moderno, como nació encima de las ruinas de la Segunda Guerra Mundial, no funciona, lo que 
se manifiesta en el surgimiento, la difusión y la persistencia de instituciones como la ronda campesina.

$\mathrm{Si}$ nos quedamos en la ronda campesina un momento, vemos a esta institución como el vehículo de defensa de la comunidad indígena en un mundo moderno sumamente hostil al mundo tradicional con sus indígenas, sus campesinos y sus comunidades. Le toca a la ronda campesina defender a los indígenas y los campesinos contra el Estado, que en su constitución se ha comprometido a defender los derechos de los ciudadanos, incluyendo a los indígenas. Solamente que al Estado se le olvidó esta obligación, pues definitivamente no es el Estado de los indígenas, ni de los campesinos ${ }^{36}$.

La ronda campesina es una institución notable, pero no es única, pues los indígenas en el Perú no son los únicos que se encuentran en la situación de defenderse contra un Estado que no los defiende. En Colombia también encontramos una institución parecida, en la guardia tribal, entre la que destaca la de los indígenas nasa en el valle del Cauca ${ }^{37}$. Una institución muy parecida la encontramos en la policía comunitaria en Guerrero en el sur de México: sus características son muy similares, y sus problemas y conflictos con el Estado también ${ }^{38}$.

Pero lo más relevante en el caso de la ronda campesina es el hecho de que, aun siendo muy claramente una institución de la comunidad indígena, la ronda campesina ha llegado a cubrir el territorio a nivel nacional, como se desprende de algunas de las cifras que han sido presentadas anteriormente. Quisiera mencionar dos detalles más acerca de la situación y las perspectivas de la ronda campesina en el Perú. En diciembre de 2012 fui invitado a participar en el III Congreso Nacional de la Ronda Campesina que se celebró en el suburbio San Juan Lurigancho en Lima. En aquella ocasión se reunió un gran número de delegados de rondas campesinas de todas las 24 regiones del país, se eligió por tercera vez una nueva mesa directiva de la organización a nivel nacional. En una conferencia que impartí en el Diplomado "Justicia y Pluralismo", que se está llevando a cabo desde 2006 en la Universidad Nacional en México (UNAM), postulé que el número de ronderos en el país podría llegar hasta dos millones. Inmediatamente después me sentí mal, pensando que hubiera exagerado, por lo que me dediqué a discutir mi postulado con ronderos y líderes de la organización de las rondas campesinas. A mi gran sorpresa no solo aceptaron mi cifra, sino algunos de ellos opinaban que el número de ronderos podría ser aún mayor. Como se mencionó, "en lo jurídico, la ronda campesina constituye todo un mundo legal paralelo al constitucional, que le obliga al gobierno intentar llevar a cabo la tarea imposible de legislar acerca de una institución que haya nacido originalmente por el incumplimiento legal del mismo gobierno. En lo político, existe en la ronda campesina también todo un mundo político paralelo en representación de lo que podemos llamar una utopía campesina o bien comunitaria, como se lleva a cabo en el movimiento comunalista en México y en otras partes del mundo"39. Que yo sepa, la ronda campesina es la única institución netamente comunitaria que ha llegado a tener presencia a nivel nacional.

La construcción de la carretera interoceánica ya va muy avanzada, y se prevé la terminación de la quinta etapa de la carretera dentro de pocos años ${ }^{40}$. Recientemente la construcción ha causado conflictos entre Ccarhuayo y la capital del distrito, Ocongate, pues las autoridades en Ocongate intentaron bloquear la construcción, con el resultado de que las instancias que dirigen la construcción se aliaron con las autoridades en Ccarhuayo, intentando captar su apoyo. Es bastante seguro que la existencia de la nueva carretera cambiará la vida, no solamente en Ccarhuayo, sino en toda la región, pero es una cuestión abierta de qué modo se hace este cambio. Ccarhuayo nunca fue una comunidad aislada, tal como alguna vez pensaban algunos antropólogos románticos, pero ahora, con la nueva carretera será menos aislada que nunca ${ }^{41}$.

\section{Referencias Citadas}

Aguirre Beltrán, Gonzalo

1991 Formas de gobierno indígena, México, UV/INI/Gobierno del Estado de Veracruz/FCE.

ALLPA, Grupo

2004 "Propuesta de modificación de la Ley No 24656, ley general de Comunidades Campesinas", en AAVV: Las comunidades campesinas en el siglo XXI. Situación actual y cambios normativos, Lima, ALLPA, 2004: 221-240.
Bernard, Jessie

1968 “Desorganización de la comunidad”, en Enciclopedia Internacional de las Ciencias Sociales, México, Aguilar, 1968, Tomo 2: 631-635.

Castañeda (s. f.).

Defensoría del Pueblo, República del Perú

2004) El reconocimiento estatal de las rondas campesinas. Compendio de normas y jurisprudencia, Lima, Documento. 
Degregori, Carlos Iván \& María Ponce Mariños

2000 "Movimientos sociales y Estado. El caso de las rondas campesinas de Cajamarca y Piura", en Carlos Iván Degregori, ed.: No hay país más diverso. Compendio de antropología peruana, Lima, IEP/PUCP/Universidad del Pacífico, 2000: 392-412.

Díaz Cruz, Rodrigo

2014 Los lugares de lo político, los desplazamientos del símbolo. Poder y simbolismo en la obra de Victor W. Turner, Barcelona/México, Gedisa/UAM Iztapalapa.

Flores Galindo, Alberto

1988 Buscando un inco. Identidad y utopía en los Andes, Lima, Ed. Horizonte.

Gluckman, Max

2009 Costumbre y conflicto en África (traducción e introducción de Leif Korsbaek), Lima, UCH/Universidad Nacional Mayor de San Marcos.

Huber, Ludwig \& Juan Carlos Guerrero

2006 Las rondas campesinas de Chota y San Marcos, Lima, PROJUR.

Huber, Ludwig

1995 Después de Dios y la Virgen está la ronda. Las rondas campesinas de Piura, IEP/IFEA, Colección Mínima Nº 31 .

Korsbaek, Leif \& Marcela Barrios Luna

2013 "La ronda campesina en el Perú. Entre el derecho y la política", Ponencia presentada en Las Jornadas Lascasianas XXIV, "Homenaje a Araceli Burguete Cal y Mayor", San Cristóbal de las Casas, Chiapas, México, noviembre de 2013.

Korsbaek, Leif \& Marcela Barrios Luna

2014 "La ronda campesina en Jaén, Perú", revista Ciencias Sociales (Universidad Nacional Mayor de San Marcos), $\mathrm{N}^{\circ}$ 1: 99-109.

Korsbaek, Leif

2008 "Los estragos de la modernización en una comunidad indígena en el Estado de México", en Juan Maestre Alfonso, Alba González Jácome y Ángel M. Casas Gragea, eds.: Nuevas rutas para el desarrollo en América Latina: Experiencias locales y globales, México, Universidad Iberooamericana, 2008: 321-344.

Korsbaek, Leif

2009 "Los peligros de la comunidad indígena y sus defensas", en la revista Ra Ximhai, Vol. 5, No 3 (septiembre-diciembre 2009): 373-385.

Korsbaek, Leif

2009 La etnografía de una comunidad matlatzinca en el estado de México. El sistema de cargos y la neoetnicidad en San Francisco Oxtotilpan, Municipio de Temsacaltepec, México D. F., Universidad Autónoma Metropolitana Iztapalapa (tesis Doctoral en Ciencias Antropológicas).

Korsbaek, Leif

2011A "No todas las rondas son comités de autodefensa y viceversa. Los tipos de rondas campesinas en el Perú", Revista Investigaciones Sociales (Universidad Nacional Mayor de San Marcos), No 26, junio de 2011: 15-39.

Korsbaek, Leif

2011B "Fundamentos legales de la ronda campesina", en José Gabriel Baeza Espejel, María Gabriela Gómez Guerrero \& Noemí Elena Ramón Silva, eds.: Pueblos indígenas: Debates y Perspectivas, Universidad Nacional Autónoma de México/PUMC, 2011: 661-706.
Korsbaek, Leif

2012 "El cambio de las instituciones comunitarias bajo el impacto del neoliberalismo", Ponencia presentada en el $54^{\circ}$ Congreso Internacional de los Americanistas, en Viena, Austria, julio 2012.

Korsbaek, Leif

2012 "Las rondas campesinas en San Marcos: un sondeo", en Journal of Ibero-American Studies (Praga, University Hradec Kralove), Vol. 4, 1-2: 96-119.

Korsbaek, Leif, Carlos Sandoval Muro \& Renato Salguero Haro 2008 "La ronda campesina en una comunidad campesina en el norte del Perú: La Toma en Cajamarca", Lima, Investigaciones Sociales (Universidad Nacional Mayor de San Marcos), $\mathrm{N}^{\circ}$ 20, Agosto de 2008: 181-198.

Krantz, Lasse

1977 "El campesino como concepto analítico", Nueva Antropología, Año 2, No 6: 87-98.

Laos Fernández, Alejandro, Pastor Paredes \& Edgardo Rodríguez 2003 "Rondando por nuestra ley", Lima, RID/SER.

Lerner, Samuel, ed.

2013 Informe final de la Comisión de la Verdady Reconciliación, Lima, Gobierno del Perú.

Machaca, Adelina

2000 "Rondas campesinas", en revista Runa, Ayaviri, Vicaría de Solidaridad de Ayaviri, $\mathrm{N}^{\circ} 43$.

Martínez Sifuentes, Esteban

2001 La policía comunitaria. Un sistema de seguridad pública comunitaria indígena en el Estado de Guerrero, México, Instituto Nacional Indígena.

Marzal, Manuel M.

1993 Historia de la antropología indigenista: México y Perú, Barcelona/México, Anthropos/UAM Iztapalapa.

Paredes Pando, Oscar

1992 Carretera Interoceánica. Integración o marginación de la Región Inka, Cusco, Centro de estudios regionales andinos "Bartolomé de las Casas".

Pérez Mundaca, José

1994 "Poder, violencia y campesinado en Cajamarca. El caso de la microrregión central", en Carlos Iván de Gregori \& al.: Perú. El problema agrario en debate, SEPIA IV: 459-485.

Polsby, Nelson W.

1968 "El estudio del poder en la comunidad", en "Enciclopedia Internacional de las Ciencias Sociales", México, Aguilar, 1968, Tomo 2: 625-631.

Ramírez Salazar, Reyder Henry

2007 Justicia ordinaria y justicia comunal en AndahuaylasApurímac. Una mirada a las instituciones locales de la administración de justicia, Lima, PROJUR.

Ricco Monge, Sergio y Leif Korsbaek

1992 "La "república" de Santiago Atitlán", revista Ojarasca, México, No 8, 1992: 49-55.

Rodríguez Aguilar, César

2007 Las rondas campesinas en el sur andino, Lima, PROJUR/ SER.

Rojas, Telmo

1990 "Rondas, poder y terror", en Alternativa, Revista de Análisis del Norte, $N^{\circ} 13$, Mayo 1990: 83-120.

Rúa S., Efraín

2005 El crimen de La Cantuta, Lima, Cuarta Edición.

Sanders, Irving T.

1968 "Desarrollo de la comunidad", en Enciclopedia Internacional de las Ciencias Sociales, México, Aguilar, 1968, Tomo 2: 635-639. 
Sandoval Forero, Eduardo

2008 La guardia indígena nasa y el arte de la resistencia pacífica, Bogotá, Fundación Hemera.

Starn, Orin

1991 "Con los llanques todo barro. Reflexiones sobre rondas campesinas, protesta rural y nuevos movimientos sociales", Lima, IEP.

Turner, Victor W.

1996 Schism and Continuity in an African Society. A Study of a Ndembu Village Life, Oxford/Washington, Berg.
Valero Moreno, Guillermo

1998 Las comunidades en el Perú. Una visión nacional desde las series departamentales, Lima, Coordinadora Rural del Perú.

Wolf, Eric R.

1966 Peasants, New Jersey, Prentice-Hall.

Zarzar, Alonso

1991 "Las rondas campesinas de Cajamarca: de la autodefensa al ¿autogobierno?”, en Luis Pásara, Rocío Valdeavellano \& Alonso Zarzar, eds.: "La otra cara de la luna. Nuevos actores sociales en el Perú", Buenos Aires, CEDYS, 1991.

\section{Notas}

1 Pido disculpas por el uso repetido de las palabras "moderno" y "tradicional", pues el recurso de la repetición no se utiliza tanto en español como en otras lenguas. Sin embargo insisto, por razones sentimentales, pues el español no es mi lengua materna y recuerdo que algunas de las primeras palabras que aprendí en español hace muchos años fueron las palabras "de vez en cuando", repetidas muchas veces en un discurso que dio Volodia Teitelboim en Copenhague en los años setenta. Pero, de manera menos sentimental, me es importante subrayar que los dos conceptos no tienen un sentido de evaluación, a diferencia de la obsesión modernizadora de los diversos gobiernos neoliberales. Mi uso de los conceptos tiene su origen e inspiración en la obra de Louis Dumont, tal como señalo en mi tesis doctoral (Korsbaek, 2009: 4-5).

2 Adelina Machaca, 2000: 9, a lo que César Rodríguez Aguilar comenta que "es incompleta porque no incorpora otras funciones que ejercen estas organizaciones, limitándolas solamente a ejercer funciones de seguridad y paz comunal" (2007: 14-15).

3 Laos, Paredes \& Rodríguez, 2003: 13.

4 Defensoría del Pueblo, 2004: 13.

5 Rojas, 1990: 89, de donde proviene también el siguiente documento citado. Firmaron legiblemente en las dos páginas seguidas del cuaderno donde está el acta, los siguientes ciudadanos: José Isael Idrogo Marín, Artidoro Huanambal, Arturo Díaz Campos, César Benavides Mejía, Aladino Burga Huanambal, Santos Saldaña Gálvez, José Oblitas C., Octavio Benavides H., Régulo Oblitas Herrera, Clodomiro Idrogo Marín (Siguen trescientas firmas).

6 Zarzar, 1991: 108. En varias otras ocasiones (entre otros lugares, en Korsbaek, 2009) he señalado que "los antropólogos somos particularmente torpes en nuestro tratamiento del factor tiempo", y creo que esta debilidad ha afectado también nuestra capacidad para captar la dinámica presente en el nacimiento de las rondas campesinas.

7 Degregori \& Ponce, 2000: 395.

8 Starn, 1991: 38

9 Yo he discutido los diversos tipos de rondas campesinas en un artículo dedicado específicamente a compararlos (Korsbaek, 2011A).

10 Rodríguez Aguilar, 2007: 17-18.

11 Huber, 1995: 121-122.

12 Huber \& Guerrero, 2006: 13.

13 Degregori \& Ponce, 2000: 405.

14 Ramírez Salazar, 2007: 25.
15 Rodríguez Aguilar, 2007: 16.

16 Rodríguez Aguilar, 2007: 17-18.

17 Información verbal de Linda Olvera Farfán, bachiller en antropología social de la Universidad Nacional San Antonio Abad del Cusco, que ha hecho trabajo de campo allá.

18 Lerner, ed., 2013: 13.

19 En realidad podemos buscar los antecedentes del modelo ya en la temprana Colonia, alrededor de 1600, en la política de congregación. En la Relación de Metepec leemos acerca de los indígenas que "aullaban como coyotes, pero se dejaron congregar".

20 Arthur Lepic, de la Red Voltaire: "Última limpieza antes de la descolonización, 1948-1960: Estado de emergencia en Malasia".

21 Citando de un breve texto del internet: "Las guerras americanas y los enfrentamientos con Europa"

22 El texto es un editorial de CHUJ WALIJO'Q, Información mensual de la Defensoría Maya, de Guatemala, $N^{\circ} 4$, de mayo 1997. De Guatemala se encuentra una narración de Santiago Atitlán, de 1990, de donde los habitantes habían logrado expulsar al ejército y a la policía judicial, después de 1.100 asesinatos durante una década, en Ricco \& Korsbaek, 1992.

23 La fuente de esta información es Castañeda, s. f.: 1-2.

24 Cuzco (que se escribe también Cusco) es una de las 24 regiones (conocidas también como departamentos) del Perú; cada región contiene un número variable de provincias, Cusco abarca 13 provincias, de las cuales Quispicanchis es, en términos de territorio, la segunda más grande, con $7.862,6 \mathrm{~km}^{2}$, en términos de población es la $4^{\text {ta }}$, con 75.900 habitantes de un poco más de un millón que es la población total de la región.

25 El censo aquí presentado proviene del centro de salud en Ccarhuayo y corresponde al 2007.

26 Korsbaek, 2012: 3.

27 Flores Galindo, 1988: 7.

28 Valero Moreno, 1998.

29 En los artículos acerca de la comunidad en la respetable y por lo general confiable Enciclopedia Internacional de las Ciencias Sociales se discute de manera pormenorizada las complicaciones relacionadas con "la investigación moderna sobre el poder en la comunidad", la "desorganización de la comunidad" y el "desarrollo de la comunidad", pero en ningún lugar encontramos una definición clara y concisa de lo que es una comunidad, y mucho menos un artículo de la misma extensión dedicado a definir el concepto (Polsby, 
1968: 625; Bernard, 1968: 631; Sanders, 1968: 635). Si nos dirigimos a la antropología propiamente, la situación no es muy diferente. En una concienzuda reseña de estudios de comunidad en Mesoamérica de toda una década, de 1968 a 1978, los autores señalan que "hasta la fecha, existen más de 100 publicaciones en forma de libro (book-length studies) de comunidades en México y Guatemala", dedicándose luego a la discusión de 149 publicaciones; y sin embargo, hay solamente una escueta definición de lo que es la "comunidad" en la reseña: "El término comunidad puede ser ambiguo. En nuestro sentido, una comunidad es un grupo de gentes en estrecha contigüidad, por lo regular en un lugar con fronteras geográficas o políticas reconocidas" (Chambers \& Young, 1979: 46), con lo que no quiero decir, de ninguna manera, que tampoco se encuentre en las 149 publicaciones reseñadas una discusión de lo que es la comunidad. Las hay, pero son contadas.

30 En otras ocasiones he explicitado qué se esconde detrás de estas seis características (notablemente en Korsbaek, 2008).

31 A menos que se señale otra cosa, la información etnográfica en el artículo proviene de mi trabajo de campo en 2007 y posteriores visitas a Ccarhuayo, con entrevistas aplicadas a ronderos, campesinos y líderes de la ronda en Ccarhuayo.

32 El problema de las características de derecho comunitario (como se llama el derecho consuetudinario en el Perú) de la ronda campesina ,y su complicada coexistencia con el derecho constitucional, es un conflicto permanente en la vida de los campesinos en el Perú, se encuentra tratado con detalle en Korsbaek, 2011B.

33 Es cierto que Perú comparte con México una característica ausencia de justicia, pero no faltan leyes. El proceso social y jurídica en las comunidades campesinas e indígena está notoriamente reglamentado por todo un conjunto de leyes, una reglamentación que define también sus órganos de gobierno local, en el caso de esas comunidades notablemente la ley no. 24656 de 1987: "en 1987, ante la ausencia de un marco legal que regulara la autonomía comunal, se aprobó la Ley General de Comunidades Campesinas, derogando el Decreto Supremo No 37-70-A" (ALLPA, 2004: 221). En esta legislación quedan bien definidas las condiciones del "gobierno local", es decir, de la comunidad; es interesante comparar con las condiciones en México, como se desprenden de un texto clásico, Formas de gobierno indígena (Aguirre Beltrán, 1991), recordando la confusión que rige en los dos países entre las definiciones de "campesinos" e "indígenas".

34 Aparte del hecho de que es una especie de "intelectual orgánico", puede ser interesante que su carrera es de pedagogía y que estudió en La Cantuta, la universidad pedagógica del Perú, pues fue uno de los centros de movimientos políticos de la izquierda (véase Rúa, 1993).

35 El siguiente cuestionario fue desarrollado para el estudio del sistema de cargos en mi tesis doctoral (Korsbaek, 2009), luego lo he adaptado para el estudio de la ronda campesina. Es importante por dos razones. En primer lugar, se ha aplicado en mi estudio de la ronda campesina en diversas partes del Perú, donde sirve para poder comparar el perfil de los ronderos en comunidades muy diferentes: en La Toma en Cajamarca (Korsbaek \& al., 2008), en San Marcos en Cajamarca (Korsbaek, 2012), en Jaén en Cajamarca (Korsbaek \& Barrios, 2014), y en un trabajo de campo que está en proceso en la comunidad quechua Corani, en Puno. En segundo lugar, como herencia de la Escuela de Manchester, alejándonos del clásico funcionalismo, sirve para proporcionarle carne y hueso a los actores en el drama social (véase al respecto Gluckman, 2009, Turner, 1996 y Díaz Cruz, 2014).

36 Los campesinos y los indígenas comparten en muchas ocasiones ese mismo destino. Acaba de salir un dossier en el no. 20 de la revista La Pacarina del Sur con una presentación de diez instituciones de defensa de la comunidad, entre indígenas y campesinos, en México, Perú, Colombia y Guatemala.

37 Véase al respecto Sandoval, 2008.

38 Véase Martínez, 2001.

39 Korsbaek \& Barrios, 2013: 27.

40 Acerca de la Carretera Interoceánica, véase Paredes, 1992.

41 El presente texto ha sido presentado en varias ocasiones en varios estados de elaboración, el antecedente inmediato es su presentación en "El Quinto Congreso sobre organización Social Tradicional" que se celebró en la Escuela Nacional de Antropología e Historia (ENAH) en agosto de 2013. La primera parte del trabajo de campo para este texto fue hecho durante mi año sabático, en 2007, con el apoyo de la Universidad Nacional de San Antonio Abad del Cusco, con varias estancias posteriores en el campo. El texto forma parte de un libro acerca de la ronda campesina en el Perú, con etnografías de cinco comunidades indígenas y campesinas, más un capítulo acerca de las condiciones en la selva amazónica y un aparato crítico-metodológico que se pretende terminar en el transcurso del 2014. Quisiera agradecer el apoyo y la ayuda brindados en la investigación por Lourdes Eliana Sánchez Báez y José Antonio Valverde, ambos bachilleres ("Pasantes", como se dice en México) en dicha Universidad; y aparte va mi agradecimiento a muchos de los habitantes en Ccarhuayo y en Ocongate, y a muchas gentes en Cusco. 
